\title{
AVASCULAR NECROSIS OF THE FEMORAL HEAD
}

\author{
An Anatomical Study* \\ T. J. Claffey, Sydney, Australia \\ From the Nuffield Orthopaedic Centre, Oxford
}

Since the recent publication of studies of the vascular pattern of the proximal femoral epiphysis in man by Tucker (1949), Trueta and Harrison (1953), Judet, Judet, Lagrange and Dunoyer (1955), and Trueta (1957) there has been a renewed interest in the mechanism of capital necrosis after fractures of the femoral neck. The literature on the subject is so extensive that no effort will be made here to quote even the most outstanding work, for the aim of this paper is to report the results of an investigation into the vascular damage caused by fractures of the femoral neck in the adult.

ANATOMY

A preliminary study by vascular injection for control material, after the method described by Trueta, Barclay, Franklin, Daniel and Prichard (1947) has provided further information on the findings of Trueta and Harrison (1953). A summary of this work must be made here because we shall repeatedly refer to the findings.

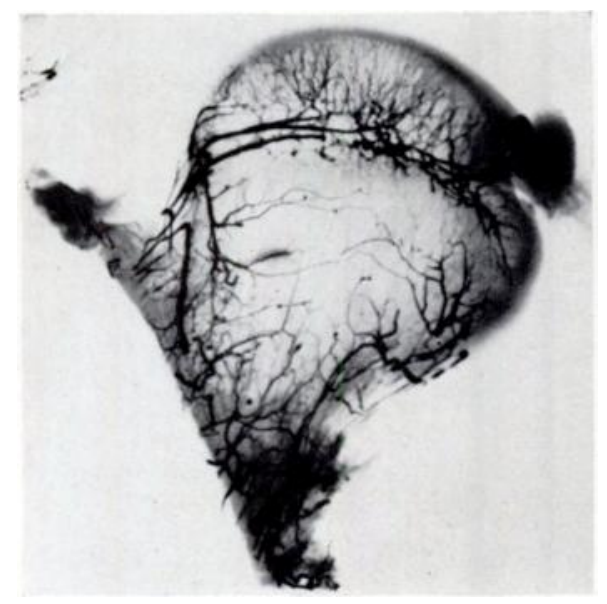

FIG. 1

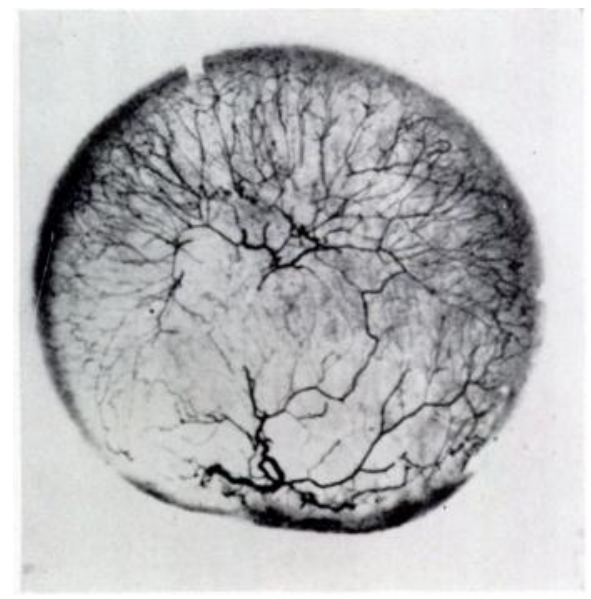

FIG. 2

Figure 1-Sagittal section of an injected femoral head, showing the lateral epiphysial vessels entering above and laterally and passing across the head in a chain to join the ligamentum teres group. Below and medially the inferior metaphysial vessels can be seen entering the bone in several different places. Figure 2-Coronal section of an injected femoral head. Above is part of the vascular chain from the lateral epiphysial vessels to the ligamentum teres. The periphery of the head is supplied in radiating fashion from this chain. Below are the inferior metaphysial vessels. (Reproduced from Trueta and Harrison, by kind permission of the Journal of Bone and Joint Surgery.)

Figure 1 shows that in the adult-even, as in this case, shortly after fusion of the growth plate-there is a rich chain of anastomoses between the lateral epiphysial vessels, the ligamentum teres group and the inferior metaphysial vessels. After epiphysial fusion free anastomosis progressively occurs between these vessels and those coming up from the neck and crossing the epiphysial scar. Figure 2 shows a transverse section of the femoral head

* This work was carried out at Oxford while the author was the holder of a Fellowship in Orthopaedic Surgery from the Post-graduate Committee in Medicine of the University of Sydney. 


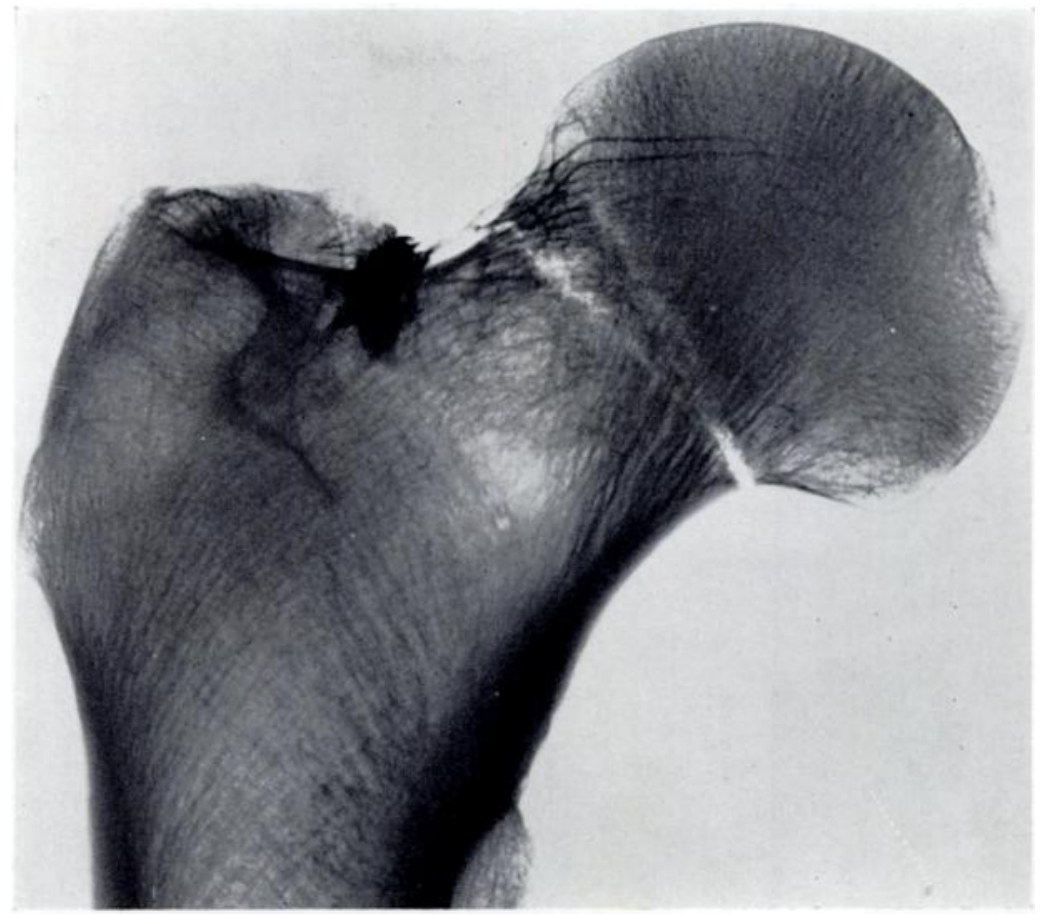

Fig. 3

The femoral neck has been divided without damage to the lateral epiphysial vessels.

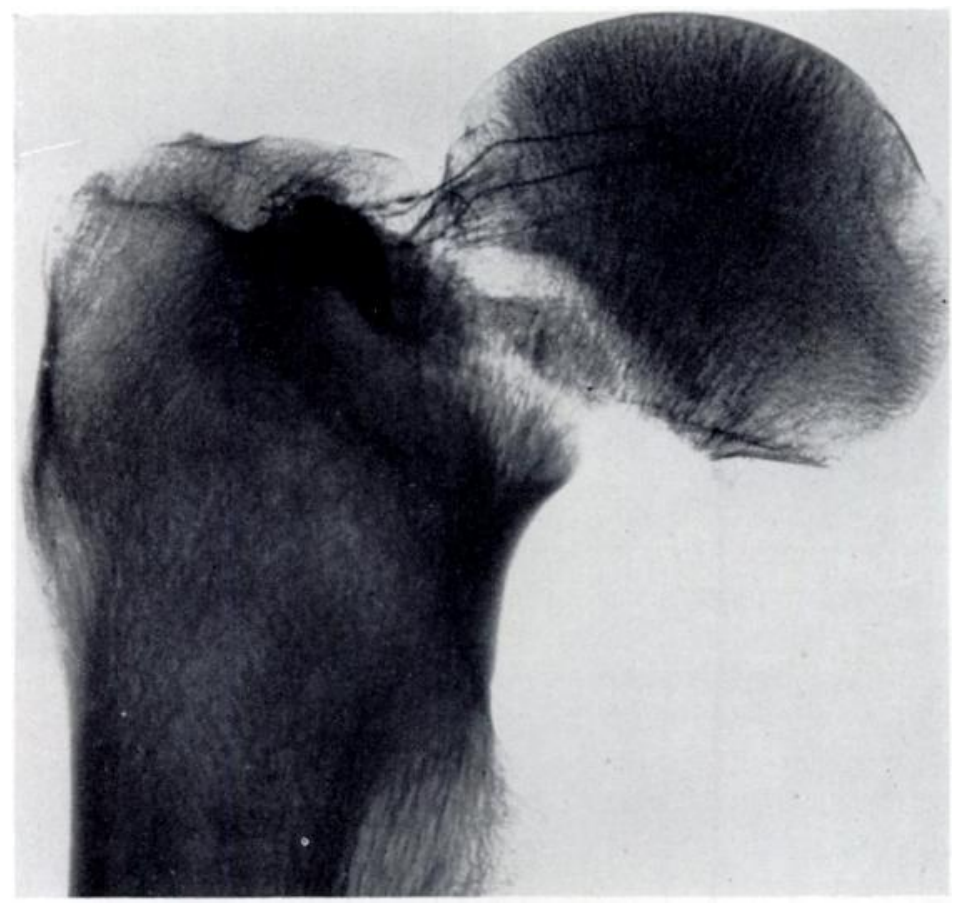

Fig. 4

Substantial lateral rotation of the distal fragment tends to relax the tension on the lateral epiphysial vessels instead of rupturing them.

VOL. 42 B, NO. 4, NOVEMBER 1960 
similarly injected. Two main groups of vessels are seen, the lateral epiphysial above and the inferior metaphysial below. The whole of the periphery of the head is supplied by arborising branches from these main vessels.

The relative importance of each group of vessels in supplying the femoral head has been studied and the part played by the fracture and by the Smith-Petersen nail in causing ischaemia of the head has been investigated.

The vessels of the ligamentum teres-It was found by Trueta and Harrison (1953) that the vessels of the ligamentum teres remained patent in the aged. This was confirmed in all the injected specimens prepared for this work. However, the fact that the vessel is patent does not prove that it is capable of keeping the head alive when all other sources of blood supply are interrupted. It was impossible, clinically, to detect whether the ligamentum teres was intact or not; consequently, attention was concentrated on the other two sources of blood, the inferior metaphysial and the lateral epiphysial vessels.

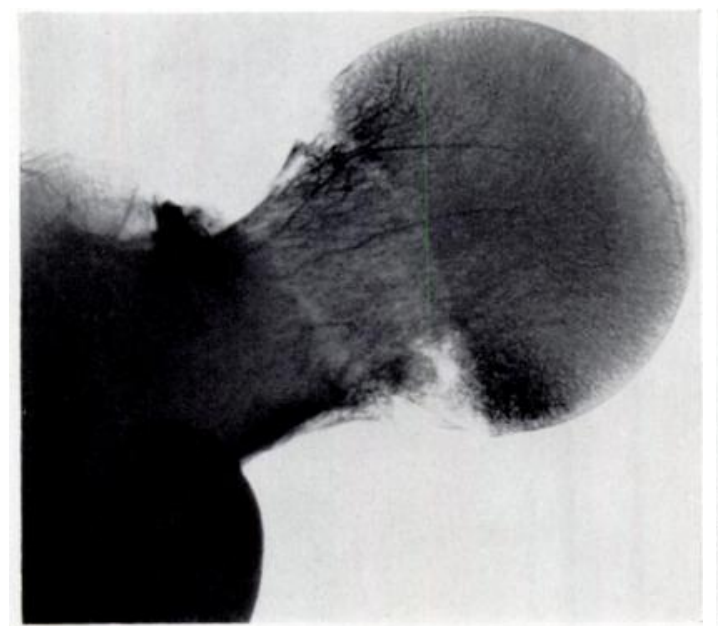

Fig. 5

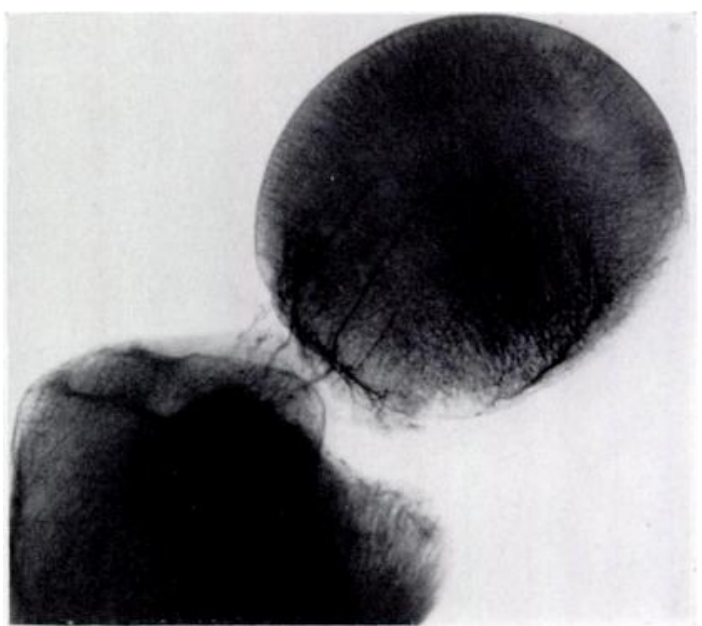

Fig. 6

Figure 5-Lateral view showing the lateral epiphysial vessels before displacement. Figure 6-After the distal fragment has been considerably displaced backwards.

Inferior metaphysial vessels-These vessels lying on the antero-inferior aspect of the femoral neck are certainly torn in all displaced fractures of the femoral neck by the lateral rotation of the distal fragment; in fractures produced experimentally this was always so. The extensive anastomosis of the inferior metaphysial vessels with the surrounding vascular groups may explain why their damage is not important.

No constant relationship between the integrity of this group of vessels and subsequent avascular necrosis could be found from radiological evidence.

The lateral epiphysial vessels-In the vascular studies on children by Trueta (1957) it was found that the lateral epiphysial vessels were responsible for the blood flow of almost the whole of the femoral capital epiphysis until the age of six to eight years and that from that age onwards the vessels from the round ligament supplied a variable segment of the medial part of the head. Trueta and Harrison (1953) found that a large anastomosis between the lateral epiphysial vessels and those of the round ligament persisted indefinitely and that the most conservative estimate made the lateral epiphysial vessels responsible for the nourishment of at least the outer two thirds of the femoral head.

In the present work we investigated afresh the outer course of the lateral epiphysial vessels before cutting the bone. 
Two important findings were made in necropsy specimens in which we caused experimental fractures after intravascular perfusion. The first observation was that a fracture distal to the point of entry of these vessels into the bone caused, as in accidental fractures in the living subject, upward displacement and lateral rotation of the distal fragment. When this happened the superior retinaculum containing the lateral epiphysial vessels stripped off the bone and a considerable displacement was possible without damage to, or tension of, the vessels (Figs. 3 to 7). Indeed, with this usual displacement the lateral epiphysial vessels were relaxed rather than stretched. It was found that only when the distal fragment was displaced proximally by a distance equal to half the diameter of the head did any tension begin to be exerted on the vessels.

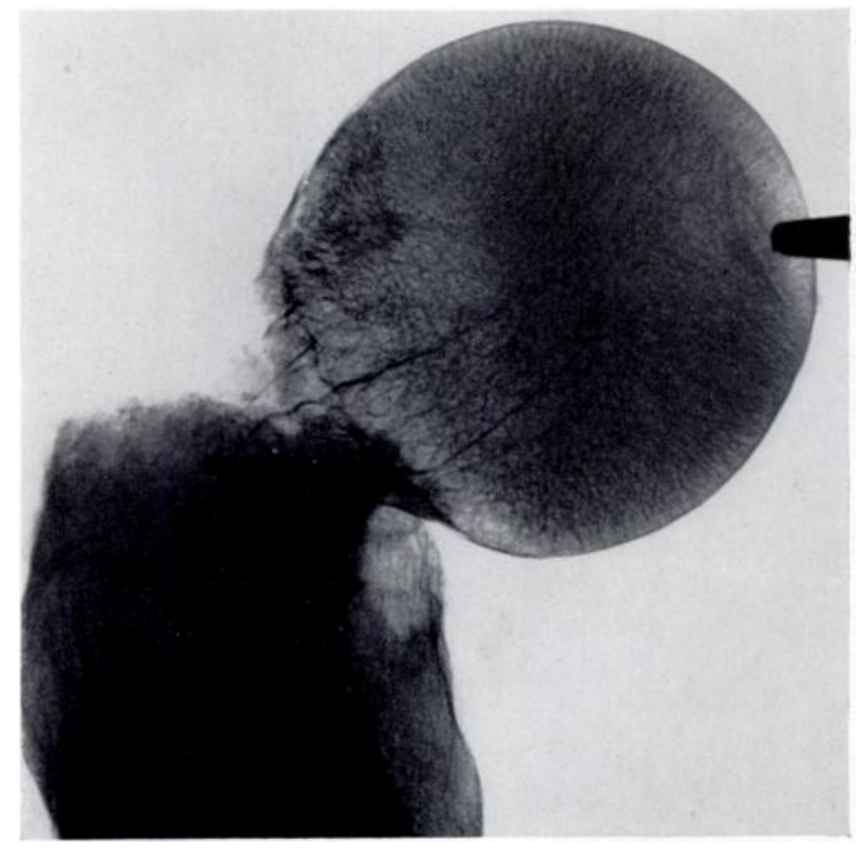

Fig. 7

Extreme rotation of the head backwards fails to damage the lateral epiphysial vessels.

The other interesting observation refers to the course of the lateral epiphysial vessels within the bone. These vessels enter the femoral head at the point where the neck turns abruptly upwards, about half a centimetre below the margin of the articular cartilage. Occasionally they enter distal to this point but seldom proximal to it. We shall refer later to the importance of these vessels in keeping the femoral head alive.

In all our specimens, when the line of fracture reached the point where the neck turned upwards, the lateral epiphysial vessels were found completely severed. This was to be expected from the work of Trueta and Harrison; it makes the determination of the line of fracture a necessity before prognosis is made.

\section{RADIOGRAPHIC STUDY OF THE FRACTURE LINE}

The pre-reduction radiographs of fractures gave no reliable information as to whether the lateral epiphysial vessels had been damaged or not, for the exact site of the fracture could not be determined until radiographs were obtained after reduction, and even then the line of fracture was often doubtful.

For this study fifty unselected radiographs after reduction of fractures of the femoral neck were examined. It was found that, of these fifty cases, in only twelve was the fracture line 
clearly seen to cross the interosseous course of the lateral epiphysial vessels. Figures 8 and 9 are two examples of this group. In none of the other cases could enough information be gathered from the radiographs about the exact site of the fracture. It was thus doubtful whether, with the present radiographic technique, the integrity or otherwise of the lateral epiphysial vessels could be determined.

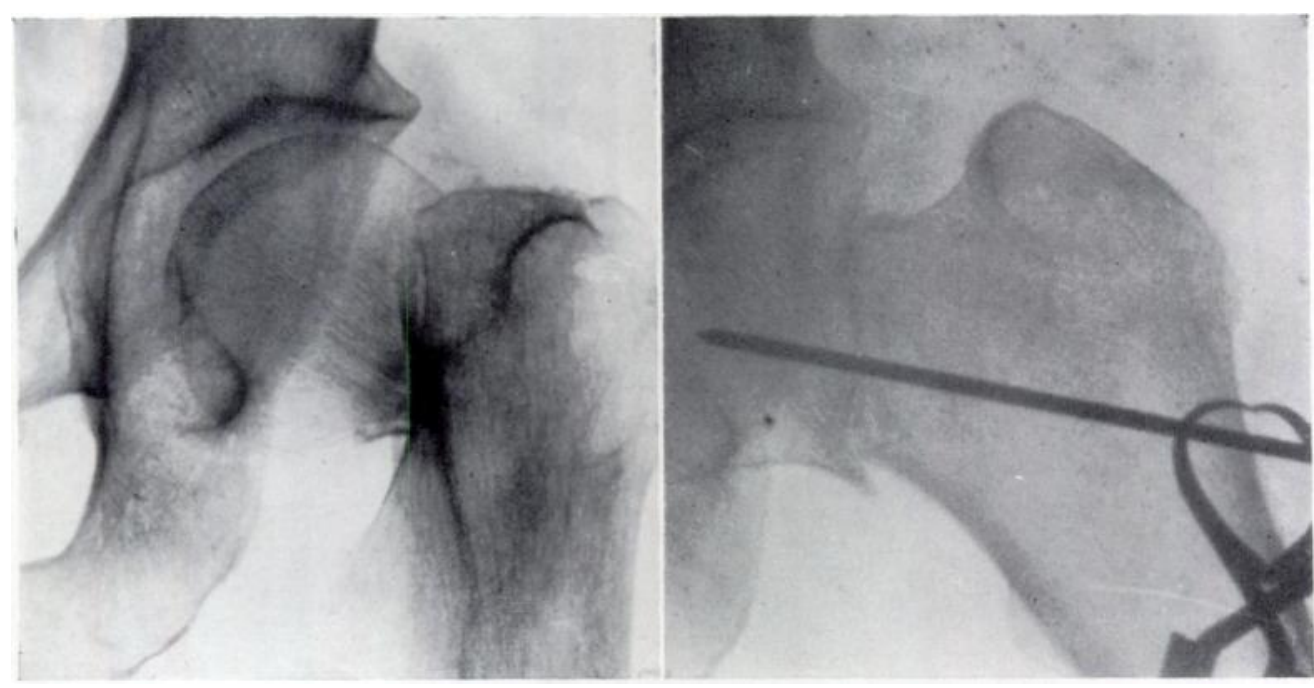

Fig. 8

Example of group 1 fracture before (left) and after (right) reduction.

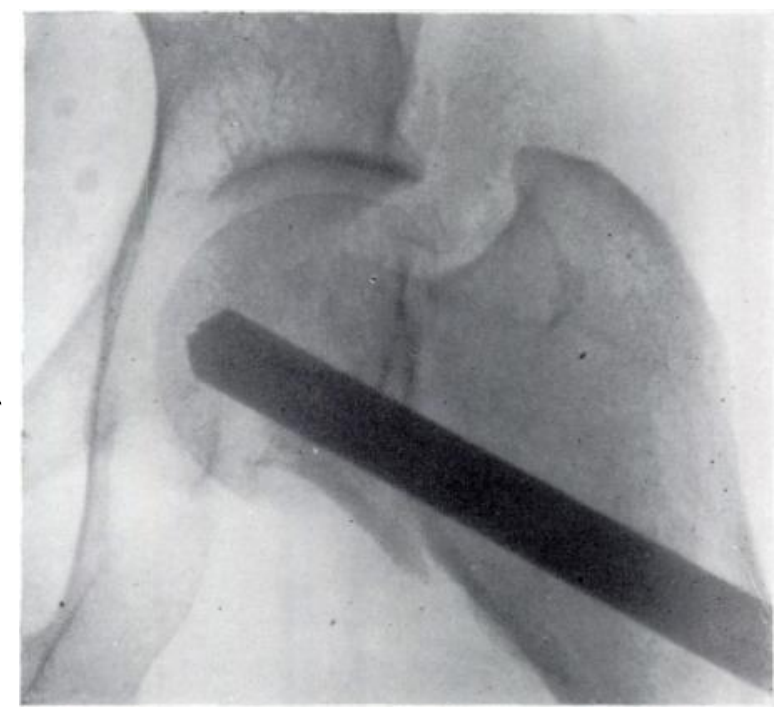

Fig. 9

Another example of group 1 fracture. In this case a loose fragment is present at the critical point. This is always a dangerous state of affairs.

\section{THE SMITH-PETERSEN NAIL}

In order to investigate the relationship between the Smith-Petersen nail and the vessels of the femoral head twelve necropsy specimens from subjects over the age of sixty-five were injected with Micropaque by the technique of Trueta and Harrison (1953). A Smith-Petersen nail was then inserted, and the specimen was radiographed, decalcified and studied in 
Spalteholz preparations. Routine radiographs gave the impression that a nail placed in the supero-lateral aspect of the head might sever the anastomosis between the lateral epiphysial vessels and those of the ligamentum teres.

Closer three-dimensional study of the Spalteholz preparations reveals an important point (Figs. 10 and 11). The nail is seen to sever one branch of the main lateral-medial anastomosis but not both. This was found in all specimens. No matter where the nail was placed it was impossible to sever completely these vascular connections. In lateral views it was seen that the width of the nail was about the same as the width of the vascular leash forming the anastomosis; so it was virtually impossible to place the nail in such a position that it could completely sever this main vascular pathway.

A nail driven through the region of the attachment of the ligamentum teres might be expected to interrupt the proximal part of the great transverse vascular anastomosis. To investigate this possibility a further six uninjected specimens were studied. A Smith-Petersen nail was placed in each case, passing deep into this part of the femoral head. The circumflex vessels on the back of the neck were then injected with a mixture of 3 per cent Micropaque and 2 per cent Berlin Blue. It was expected that the flow through the ligamentum teres group would be stopped if the nail had severed the anastomotic chain. The result was the same in all instances. The dye passed along the anastomosis and emerged from the cut end of the ligamentum teres.

These findings suggest that it is almost impossible to interrupt seriously the anastomosis between the vessels of the lateral epiphysial group and those of the ligamentum teres with a Smith-Petersen nail. It seems most unlikely therefore that the nail contributes greatly to avascular necrosis of the femoral head.

\section{THE ROLE OF THE LATERAL EPIPHYSIAL VESSELS IN AVASCULAR NECROSIS}

Clinical evidence-Consideration of these observations suggested that the most likely cause of avascular necrosis of the femoral head was damage to the lateral epiphysial vessels. To collect further information on this point records of the femoral neck fractures seen in 1950-55 in the Accident Service of the Nuffield Orthopaedic Centre at the Radcliffe Infirmary were studied. These were classified by their radiographic appearance into two groups: 1) those in which the fracture line undoubtedly reached the point of entry of the lateral epiphysial vessels; and 2) all the other cases.

Of a total of 197 patients 178 could be traced. The radiographs of twenty-four of these clearly belonged to group 1. In all of them avascular necrosis of the femoral head occurred. Of a further group of twenty cases in which the radiographs were not clear enough to disclose the integrity or otherwise of the area of the lateral epiphysial vessels, ten suffered from necrosis of the head.

\section{DISCUSSION}

It was impossible from this investigation to ascertain whether damage to the vessels of the round ligament played any part in the causation of capital necrosis. The fact that necrosis occurred in all the cases in which the fracture line crossed the area of penetration of the lateral epiphysial vessels suggests that the damage to these vessels is the most important single factor responsible for necrosis of the femoral head.

The finding of the part played by these vessels in the prognosis of femoral neck fractures may suggest a new interpretation to the observation by Pauwels (1935) that the more vertical was a fracture of the femoral neck the less likely was it to end successfully. That the vertical fractures are more unstable and more likely to slip seems obvious. However, this can bear only on the rate and quality of consolidation but not on the vascular integrity of the femoral head. Figure 12 shows schematically that an oblique or transverse fracture can interfere with 


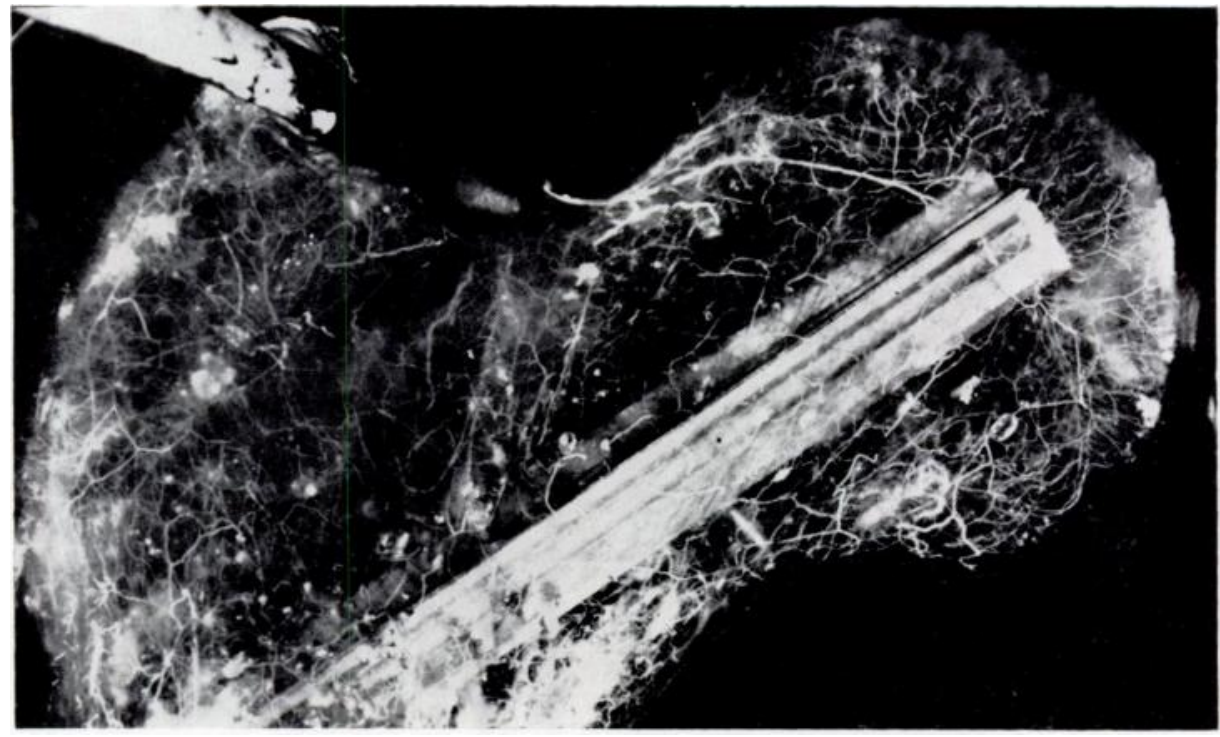

FIG. 10

Spalteholz preparation of an injected femoral head and neck viewed from in front. A nail has been inserted, and one fin can be seen cutting through the vascular chain.

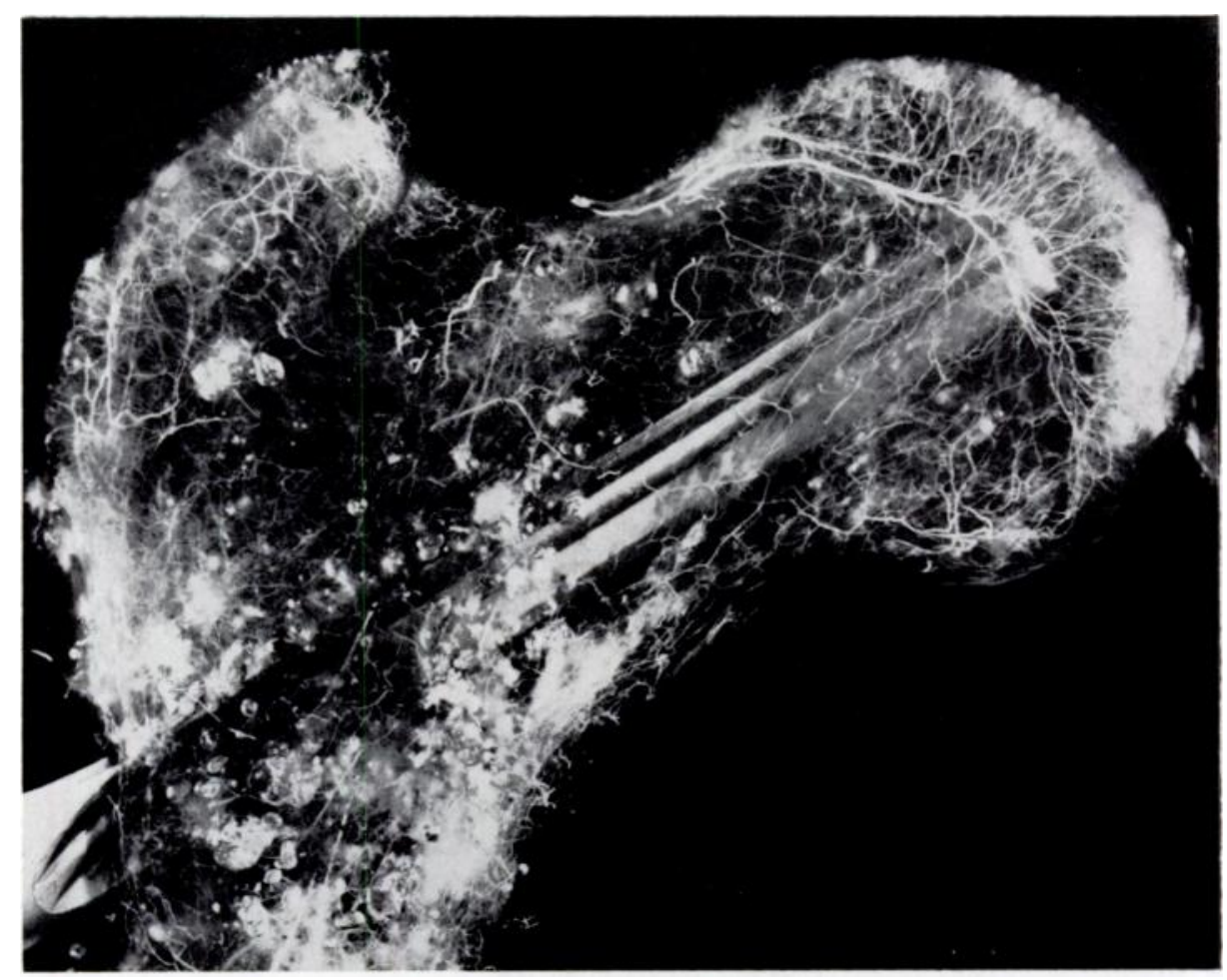

Fig. 11

Same specimen as in Figure 10 viewed from behind. Note that the posterior part of the vascular chain is intact. 
the lateral epiphysial vessels as much as a vertical fracture if it is placed at the proximal end of the neck.

Two other observations made are worth emphasising. One, which we found surprising, was that displacement of the distal fragment hardly damaged the lateral epiphysial vessels. The other observation was the limited part that the nail plays in causing vascular interference sufficient to endanger the vitality of the femoral head.

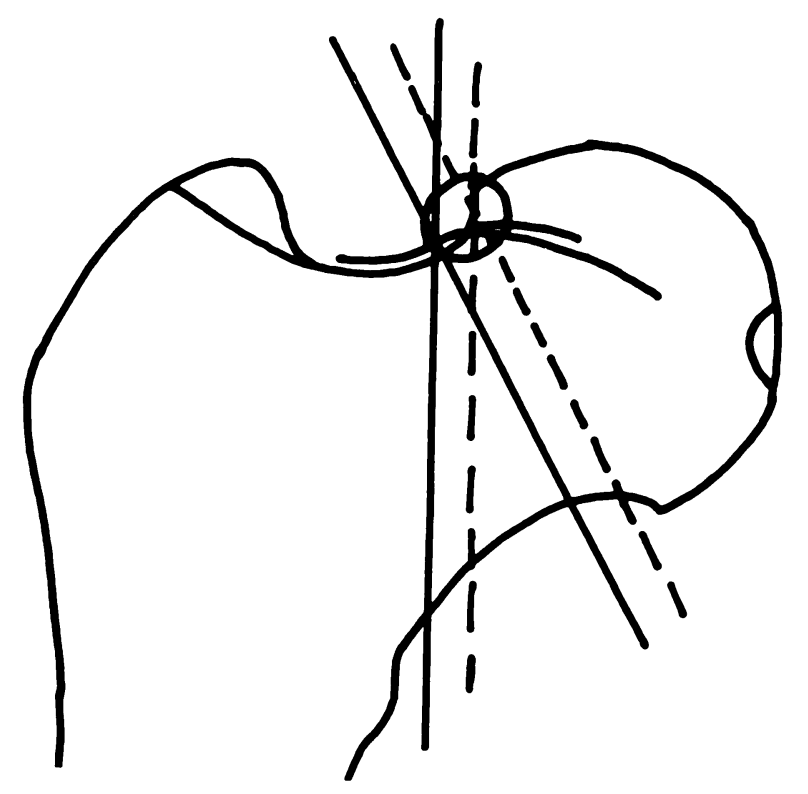

Fig. 12

The interrupted lines represent group 1 fractures of varying obliquity.

It may be concluded from this work that determination of the line of fracture gives the best indication of the fate of the femoral head.

$\mathrm{I}$ am indebted to Professor $\mathrm{J}$. Trueta for his inspiration and guidance in this work. The clinical material from the Accident Service of the Radcliffe Infirmary was made available by the kind permission and cooperation of Mr J. C. Scott. To him and his colleagues, Mr R. G. Taylor and Mr J. D. Morgan, I express my gratitude. I also thank Mr A. Mann and Mr D. W. Charles of the technical staff of the Nuffield Department of Orthopaedic Surgery for their help.

\section{REFERENCES}

Judet, J., Judet, R., Lagrange, J., and Dunoyer, J. (1955): A Study of the Arterial Vascularization of the Femoral Neck in the Adult. Journal of Bone and Joint Surgery, 37-A, 663.

Pauwels, F. (1935): Der Schenkelhalsbruch, ein mechanisches Problem. Stuttgart: Enke.

Trueta, J. (1957): The Normal Vascular Anatomy of the Human Femoral Head During Growth. Journal of Bone and Joint Surgery, 39-B, 358.

Trueta, J., Barclay, A. E., Franklin, K. J., Daniel, P. M., and Prichard, M. M. L. (1947): Studies of the Renal Circulation. Oxford: Blackwell Scientific Publications.

Trueta, J., and Harrison, M. H. M. (1953): The Normal Vascular Anatomy of the Femoral Head in Adult Man. Journal of Bone and Joint Surgery, 35-B, 442.

TUCKer, F. R. (1949): Arterial Supply to the Femoral Head and its Clinical Importance. Journal of Bone and Joint Surgery, 31-B, 82.

VOL. 42 B, No. 4, NOVEMBER 1960 